PROCEEDINGS OF THE

AMERICAN MATHEMATICAL SOCIETY

Volume 30, Number 2, October 1971

\title{
ON CATEGORIES OF QUOTIENTS
}

\section{AARON KLEIN}

ABSTRACT. We construct a category of quotients over a category satisfying a condition similar to the Ore condition. Addition of quotients is briefly discussed.

In a previous paper [1] a theory of relations in categories of a general type was introduced. In trying to adapt the elegant approach of Hilton [2] to the nonabelian case, a difficulty is encountered, but Hilton's method works in the associative case, in which by [1] an Ore condition holds. In this case a generalization is given which seems to be of interest; it describes a construction of a category which is algebraically a sort of category of quotients. Finally, addition is briefly discussed. Notations and definitions of [1] are freely used.

Let $\mathcal{C}$ be a finitely complete [3] bicategory [4] with classes of monics $\mathscr{I}$ and epics $\mathcal{S}$. In [1] we have constructed a near-category Re with class of objects $\left|R_{\mathfrak{e}}\right|=|\mathfrak{e}|$ and with relations as morphisms. We employ the method of Hilton [2] in the nonabelian case, but we have to use pairs of coinitial morphisms.

With fixed objects $A, B$ in $\mathcal{C}$ consider the collection $\mathcal{P}(A, B)$ of pairs of morphisms $(\alpha, \beta), A \stackrel{\alpha}{\longleftarrow} \underset{\sim}{\rightarrow} B$, and declare $(\alpha, \beta) \sim\left(\alpha^{\prime}, \beta^{\prime}\right)$ in $\odot(A, B)$ if and only if there are $\sigma, \sigma^{\prime}$ in $\mathcal{S}$ that satisfy $\alpha \sigma=\alpha^{\prime} \sigma^{\prime}$, $\beta \sigma=\beta^{\prime} \sigma^{\prime}$.

In [1] we have proved that the extension $\mathcal{R}_{\mathfrak{e}}$ of $\mathfrak{C}$ is a category if and only if a condition denoted (A) holds, and that condition reads as follows: For every $\xi, \eta$ in $\mathcal{C}$ with common codomain and with $\xi \in \mathcal{S}$ there is a common right multiple $\xi u=\eta v$ with $v \in \mathcal{S}$.

I. If (A) holds in $\mathrm{e}$ then $\sim$ is an equivalence in $\mathcal{P}(A, B)$.

For, if $(\alpha \sigma, \beta \sigma)=\left(\alpha^{\prime} \sigma^{\prime}, \beta^{\prime} \sigma^{\prime}\right)$ and $\left(\alpha^{\prime} \tau, \beta^{\prime} \tau\right)=\left(\alpha^{\prime \prime} \tau^{\prime}, \beta^{\prime \prime} \tau^{\prime}\right)$ with $\sigma^{\prime} s$ and $\tau^{\prime}$ 's in $\mathcal{S}$ then, by $(\mathbf{A})$ and the pullback condition, there are $\phi, \psi \in \mathcal{S}$ such that $\sigma^{\prime} \phi=\tau \psi$; hence $\tau^{\prime} \psi, \sigma \phi \in \mathcal{S}$ and $(\alpha \sigma \phi, \beta \sigma \phi)=\left(\alpha^{\prime \prime} \tau^{\prime} \psi, \beta^{\prime \prime} \tau^{\prime} \psi\right)$.

If (A) holds we denote $\mathrm{Z}(A, B)=\boldsymbol{P}(A, B) / \sim$. Representatives for elements of $\mathrm{Z}(A, B)$ can be chosen according to: $(\alpha, \beta) \sim\left(\alpha^{\prime}, \beta^{\prime}\right)$ in $\mathcal{P}(A, B)$ if and only if the $g$-parts in $i$-s-factorizations of $\{\alpha, \beta\}$ and $\left\{\alpha^{\prime}, \beta^{\prime}\right\}$, namely $\{\alpha, \beta\} i,\left\{\alpha^{\prime}, \beta^{\prime}\right\}^{i}$ are equivalent monics. (For, if $\{\alpha, \beta\}=\{\xi, \eta\} \zeta$ and $\left\{\alpha^{\prime}, \beta^{\prime}\right\}=\{\xi, \eta\} \zeta^{\prime}$ with $\zeta, \zeta^{\prime} \in \mathcal{S}$ then there are

Received by the editors March 31, 1970.

AMS 1969 subject classifications. Primary 1810.

Key words and phrases. Category of quotients, the Ore condition, relations in categories, bicategory, near-category, category with addition, Fitting multiplication. 
$\phi, \phi^{\prime} \in \mathcal{S}$ satisfying $\zeta \phi=\zeta^{\prime} \phi^{\prime}$, hence $(\alpha \phi, \beta \phi)=\left(\alpha^{\prime} \phi^{\prime}, \beta^{\prime} \phi^{\prime}\right)$. The converse follows from the property of $i$-s-factorization.)

We denote the equivalence class of $(\alpha, \beta)$ by $\beta / \alpha$. Then if $\sigma \in \mathcal{S}$ and $\alpha \sigma, \beta \sigma$ are defined, we have $\beta / \alpha=\beta \sigma / \alpha \sigma$.

For $\beta / \alpha \in \mathcal{Z}(A, B)$ and $\gamma / \beta^{\prime} \in \mathcal{Z}(B, C)$ we define $\left(\gamma / \beta^{\prime}\right)(\beta / \alpha)=\gamma \psi / \alpha \phi$ where $\downarrow \psi, \phi, \beta^{\prime}, \beta \downarrow$ is a pullback. This composition is well defined (general proof later (IV)), it is associative and the classes $1 / 1$ are identities.

II. $Z$ is a category and the mapping $\alpha \rightarrow \alpha / 1$ is a covariant embedding of $\mathfrak{C}$ into $\mathrm{Z}$.

The mapping $\alpha \rightarrow \alpha / 1$ is one-to-one: if $\alpha / 1=\beta / 1$ then $\alpha \sigma=\beta \sigma^{\prime}$, $1 \sigma=1 \sigma^{\prime}$ with $\sigma, \sigma^{\prime} \in S$ and, since $S$ consists of epics only, this implies $\alpha=\beta$.

An isomorphism between this $Z$ and the category $R_{e}$ constructed in [1] is given by assigning to $\beta / \alpha$ of $Z$ the relation $[\cdot, \xi, \eta]$ with $\{\xi, \eta\}=\{\alpha, \beta\}^{i}$.

However, this method does not enable us to construct relations in the general case. But in the (A)-case it is considerably generalized as follows.

III. Let $\mathfrak{e}$ be a category with pullbacks (products not required). Let $\mathscr{D}$ be any subcategory of $\mathcal{C}$ with $|\mathscr{D}|=|\mathfrak{C}|$ and assume the following condition holds:

(A) $)_{D}$ : If $\downarrow v, u, \eta, \xi \downarrow$ is a pullback in $\mathcal{C}$ and if $\xi \in \mathbb{D}$ then $v \in \mathbb{D}$. (We require (A) to hold in pullbacks since we do not assume that $x y \in \mathbb{D}$ implies $x \in D$.) For $A, B \in|\mathfrak{C}|$ denote by $P^{\mathscr{D}}(A, B)$ the collection of pairs $(\alpha, \beta)$,

$$
A \stackrel{\alpha}{\leftarrow} X \stackrel{\beta}{\rightarrow} B .
$$

We declare $(\alpha, \beta) \sim_{D}\left(\alpha^{\prime}, \beta^{\prime}\right)$ in $\rho^{D}(A, B)$ if there are $\delta, \delta^{\prime}$ in $D$ satisfying $(\alpha \delta, \beta \delta)=\left(\alpha^{\prime} \delta^{\prime}, \beta^{\prime} \delta^{\prime}\right)$. Then: $\sim_{D}$ is an equivalence in $\rho^{D}(A, B)$. For, reflexivity follows from $|\mathfrak{C}|=|\mathbb{D}|$, symmetry is obvious, transitivity is similar to $\mathrm{I}$.

We denote $R^{D}(A, B)=P^{D}(A, B) / \sim_{D}$ and $\beta / \alpha=$ the equivalence class of $(\alpha, \beta)$ (more precisely $\left.\mathcal{R}_{\mathfrak{e}}^{D}(A, B), \beta / D \alpha\right)$.

IV. Let $\beta / \alpha \in \mathbb{R}^{D}(A, B), \gamma / \beta^{\prime} \in \mathbb{R}^{D}(B, C)$; if $\downarrow \psi, \phi, \beta^{\prime}, \beta \downarrow$ then $\gamma \psi / \alpha \phi$ depends only on the equivalence classes $\beta / \alpha$ and $\gamma / \beta^{\prime}$.

PROOF. Let $(\alpha \delta, \beta \delta)=\left(\alpha_{1} \delta_{1}, \beta_{1} \delta_{1}\right)$ and $\left(\beta^{\prime} \epsilon, \gamma \epsilon\right)=\left(\beta_{1}^{\prime} \epsilon_{1}, \gamma_{1} \epsilon_{1}\right)$, with $\delta$ 's and $\epsilon$ 's in $D$. We construct pullbacks

$$
\downarrow b, a, \phi, \delta \downarrow, \quad \downarrow c, d, \psi, \epsilon \downarrow, \quad \downarrow y, x, c, b \downarrow .
$$

Then $b, c, x, y \in \mathbb{D}$ by $(\mathbf{A})_{\mathfrak{D}}$. By juxtaposition of pullbacks, we obtain 
$\downarrow d y, a x, \beta^{\prime} \epsilon, \beta \delta \downarrow$. The same process with the 1 -indexed morphisms yields $\downarrow d_{1} y_{1}, a_{1} x_{1}, \beta_{1}^{\prime} \epsilon_{1}, \beta_{1} \delta_{1} \downarrow$ and $b_{1}, c_{1}, x_{1}, y_{1} \in D$. By the uniqueness of pullbacks there is an invertible $\iota$ that satisfies $a x=a_{1} x_{1} \iota, d y=d_{1} y_{1} \iota$. Therefore,

$$
(\alpha \phi)(b x)=\left(\alpha_{1} \phi_{1}\right)\left(b_{1} x_{1} \iota\right), \quad(\gamma \psi)(b x)=\left(\gamma_{1} \psi_{1}\right)\left(b_{1} x_{1} \iota\right) .
$$

Since $\iota \in D$ by $(\mathbf{A})_{D}\left(\downarrow \iota, 1, \iota^{-1}, 1 \downarrow\right.$ and $\left.1 \in D\right)$, we conclude

$$
(\alpha \phi, \gamma \psi) \sim\left(\alpha_{1} \phi_{1}, \gamma_{1} \psi_{1}\right)
$$

Now we define composition by $\left(\gamma / \beta^{\prime}\right)(\beta / \alpha)=\gamma \psi / \alpha \phi$ where $\downarrow \psi, \phi, \beta^{\prime}, \beta \downarrow$.

$\mathrm{V}$. THEOREM. $R_{e}^{D}$ with the above composition is a category with $\left|R_{\mathfrak{e}}^{D}\right|=|\mathfrak{C}|$. The identity of $A \in|\mathfrak{e}|$ in $R_{\mathfrak{e}}^{\mathbb{D}}$ is $1_{A} / 1_{A}$. The mapping $\alpha \rightarrow \alpha / 1$ of $\mathcal{C}$ into $R_{\mathfrak{e}}^{\mathbb{D}}$ is a covariant functor, and it is an embedding if and only if $D$ consists of epics only.

Proof. Associativity of composition follows by juxtaposition of pullbacks. The properties of the mapping $\alpha \rightarrow \alpha / 1$ follow by using pullbacks of simple forms. The last statement: if $\delta \in \mathbb{D}$ is not epic then there exist $\alpha, \beta$ such that $\alpha \delta=\beta \delta$ and $\alpha \neq \beta$, yet $\alpha / 1=\beta / 1$ by definition of $\sim$ D. The other part of the proof is similar to II.

Denoting $(\beta / \alpha)^{-}=\alpha / \beta$ we have an involution on $\Omega^{D}$ and the factorization $\beta / \alpha=(\beta / 1)(\alpha / 1)^{-}$. If all morphisms in $D$ are epic then we may identify $\alpha \in \mathcal{C}$ with $\alpha / 1$ in $R^{D}$, and $\Omega^{D}$ is a sort of "category of right-quotients" over $\mathcal{C}$ (see $[1,2.1]$ ). Moreover, if $D$ satisfies an extra condition " $x y \in \mathbb{D}$ and $y \in \mathbb{D}$ imply $x \in \mathscr{D}$," then $D$ consists exactly of the right-regular elements, namely $\alpha \alpha^{-}=1$ if and only if $\alpha \in \mathbb{D}$. To prove this we first observe that for $\alpha \in \mathbb{D}$ we have $\alpha \alpha^{-}$ $=\alpha / \alpha=1 / 1$. The converse: if $(\alpha, \alpha) \sim(1,1)$ then $(\alpha \delta, \alpha \delta)=\left(\delta^{\prime}, \delta^{\prime}\right)$ with $\delta, \delta^{\prime} \in D$; hence $\alpha \delta, \delta \in \mathbb{D}$, so $\alpha \in \mathscr{D}$.

If $D$ does not satisfy the extra condition, then there are $x, y$ such that $x y, y \in D, x \notin D$, but then by definition of $\sim_{\mathscr{D}}$ we have $(x, x)$ $\sim(1,1)$ (since $(x y, x y)=(1 x y, 1 x y)$ ) thus $x x^{-}=1$ and $x \notin D$.

We remark that even in the general case (nonepic $D$ ) we have $\alpha^{-} \alpha=1$ if and only if $\alpha$ is monic.

If $\mathcal{C}$ is a category with pullbacks then the subcategory of isomorphisms and $\mathcal{C}$ itself are the two extremes for which (A) holds. With the first the functor $\alpha \rightarrow \alpha / 1$ is an embedding and only the isomorphisms of $\mathcal{e}$ have the property $x x^{-}=1$; even if $x$ is a retraction we have $x y=1$ but not $x x^{-}=1$. (In the general case $x^{-}$is in the image of $\mathfrak{C}$, namely of the form $y / 1$, iff $x$ is a coretraction.) 
The functor $\mathfrak{C} \rightarrow \mathfrak{R}_{\mathfrak{e}}^{\mathfrak{e}}$ is generally not an embedding. All the elements of $\mathfrak{C}$ will have the property $x x^{-}=1$ in $\mathbb{R}_{\mathfrak{e}}^{\mathfrak{e}}$.

An intermediate category with (A) is the category of monics in $\mathfrak{C}$ and in this case we generally do not have an embedding. The monics of $\mathfrak{C}$ have invertible images by the mapping $\alpha \rightarrow \alpha / 1$.

An obvious example is the following. Let $\mathcal{C}$ be the multiplicative semigroup of positive integers (or nonzero integers). We take $\mathfrak{D}=\mathfrak{C}$ and we are in the epic case; the category $R^{D}$ is the group of positive (nonzero) rational numbers.

Let $G: \mathcal{C} \rightarrow Q$ be a functor and $a$ a category with an involution $(-)^{*}$. We ask about functors $\tilde{G}$ commuting with the involutions $\left(\widetilde{G}(\beta / \alpha)=\widetilde{G}(\alpha / \beta)^{*}\right)$ and for which the following triangle is commutative

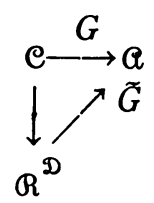

Since $\beta / \alpha=(\beta / 1)(\alpha / 1)^{-}$, we must have

$$
\tilde{G}(\beta / \alpha)=\tilde{G}(\beta / 1) \tilde{G}(\alpha / 1)^{*}=(G \beta)(G \alpha)^{*} .
$$

For $\delta \in D$ we have $\delta / \delta=1 / 1$ so $(G \delta)(G \delta)^{*}$ must be an identity. If this is the case then $G$ is well defined since for $\beta / \alpha=\beta^{\prime} / \alpha^{\prime}$ we have $(\alpha \delta, \beta \delta)=\left(\alpha^{\prime} \delta^{\prime}, \beta^{\prime} \delta^{\prime}\right)$, so

$$
\begin{aligned}
\widetilde{G}(\beta / \alpha) & =(G \beta)(G \alpha)^{*}=(G \beta)(G \delta)(G \delta)^{*}(G \alpha)^{*}=G(\beta \delta) G(\alpha \delta)^{*} \\
& =G\left(\beta^{\prime} \delta^{\prime}\right) G\left(\alpha^{\prime} \delta^{\prime}\right)^{*}=G\left(\beta^{\prime} / \alpha^{\prime}\right) .
\end{aligned}
$$

If $\downarrow \psi, \phi, \beta, \alpha \downarrow$ then $(1 / \beta)(\alpha / 1)=\psi / \phi$. So

$$
(G \beta)^{*} G(\alpha)=\widetilde{G}(1 / \beta) \widetilde{G}(\alpha / 1)=\widetilde{G}(\psi / \phi)=\widetilde{G}\left((\psi / 1)(\phi / 1)^{-}\right)=(G \psi)(G \phi)^{*} \text {. }
$$

Moreover, this condition is sufficient for $\widetilde{G}$ to be a functor. Given $\beta / \alpha, \gamma / \beta^{\prime}$ and $\downarrow \psi, \phi, \beta^{\prime}, \beta \downarrow$ we have $\left(\gamma / \beta^{\prime}\right)(\beta / \alpha)=\gamma \psi / \alpha \phi$; hence

$$
\begin{aligned}
\tilde{G}\left[\left(\gamma / \beta^{\prime}\right)(\beta / \alpha)\right] & =\widetilde{G}(\gamma \psi / \alpha \phi)=G(\gamma \psi) G(\alpha \phi)^{*}=(G \gamma)(G \psi)(G \phi)^{*}(G \alpha)^{*} \\
& =(G \gamma)\left(G \beta^{\prime}\right)^{*}(G \beta)(G \alpha)^{*}=\tilde{G}\left(\gamma / \beta^{\prime}\right) \tilde{G}(\beta / \alpha) .
\end{aligned}
$$

This concludes the proof of

VI. $\widetilde{G}$ with the properties stated above exists if and only if $(G \delta)(G \delta)^{*}=1$ for every $\delta \in \mathbb{D}$ and $(G \beta)^{*}(G \alpha)=(G \psi)(G \phi)^{*}$ for every pullback $\downarrow \psi, \phi, \beta, \alpha \downarrow$ in $\mathcal{C}$. 
We say that $\mathfrak{C}$ is a category with addition if for some pairs of objects $A, B$ a partial operation + is defined on $\mathcal{C}(A, B)$ which is distributive in the following sense: if $\alpha, \beta \in \mathcal{C}(A, B)$ and $\alpha+\beta$ is defined, then for every $\gamma: B \rightarrow C, \delta: D \rightarrow A, \gamma \alpha+\gamma \beta$ and $\alpha \delta+\beta \delta$ are defined and $\gamma(\alpha+\beta)$ $=\gamma \alpha+\gamma \beta,(\alpha+\beta) \delta=\alpha \delta+\beta \delta$. (A known example of addition is the Fitting multiplication of morphisms in group theory.)

Let $\mathcal{C}$ be a category with pullbacks and $D$ a subcategory with $|\mathfrak{D}|=|\mathbb{C}|$ and $(\mathbf{A})_{D}$. Let $\beta / \alpha, \beta^{\prime} / \alpha^{\prime} \in \mathbb{R}^{D}(A, B)$ and consider a pullback $\downarrow \psi, \phi, \alpha^{\prime}, \alpha \downarrow$. If $\beta \phi+\beta^{\prime} \psi$ is defined in $\mathcal{e}$ then we define

$$
\beta / \alpha+\beta^{\prime} / \alpha^{\prime}=\left(\beta \phi+\beta^{\prime} \psi\right) / \theta
$$

where $\theta=\alpha \phi=\alpha^{\prime} \psi$.

For instance if $\alpha$ is monic and $\beta+\beta^{\prime}$ is defined, then $\beta / \alpha+\beta^{\prime} / \alpha^{\prime}$ is defined and $=\left(\beta+\beta^{\prime}\right) / \alpha$.

VII. + is well defined in $\Omega^{D}$ and it extends the addition in $\mathrm{C}$.

Proof. If $b / a=\beta / \alpha$ and $b^{\prime} / a^{\prime}=\beta^{\prime} / \alpha^{\prime}$ then $(\alpha \sigma, \beta \sigma)=(a \tau, b \tau)$ and $\left(\alpha^{\prime} \sigma^{\prime}, \beta^{\prime} \sigma^{\prime}\right)=\left(a^{\prime} \tau^{\prime}, b^{\prime} \tau^{\prime}\right)$ with $\sigma, \sigma^{\prime} \tau, \tau^{\prime} \in \mathcal{D}$. Now consider the following pullbacks

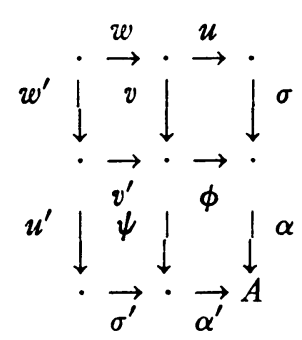

so by $(A)_{D}$ we have $\beta \phi+\beta^{\prime} \psi v w$ defined and

$$
\begin{aligned}
\beta \phi v w+\beta^{\prime} \psi v w & =\beta \phi v w+\beta^{\prime} \psi v^{\prime} w^{\prime}=\beta \sigma u w+\beta^{\prime} \sigma^{\prime} u^{\prime} w^{\prime} \\
& =(b \tau) u w+\left(b^{\prime} \tau^{\prime}\right) u^{\prime} w^{\prime} .
\end{aligned}
$$

By the extended definition of + and, considering the big pullback, we have $b \tau / a \tau+b^{\prime} \tau^{\prime} / a^{\prime} \tau^{\prime}$ defined and $=\left(\beta \phi v w+\beta^{\prime} \psi v^{\prime} w^{\prime}\right) / \eta$ where $\eta=\alpha \sigma u w=\alpha^{\prime} \sigma^{\prime} u^{\prime} w^{\prime}$. But $\tau, \tau^{\prime} \in \mathcal{D}$, hence $b \tau / a \tau=b / a$ and $b^{\prime} \tau^{\prime} / a^{\prime} \tau^{\prime}$ $=b^{\prime} / a^{\prime}$, so, since $w, v \in \mathscr{D}$, we conclude

$$
\begin{aligned}
b / a+b^{\prime} / a^{\prime} & =\left(\beta \phi v w+\beta^{\prime} \psi v w\right) / \alpha \phi v w=\left(\beta \phi+\beta^{\prime} \psi\right) v w / \alpha \phi v w \\
& =\left(\beta \phi+\beta^{\prime} \psi\right) / \alpha \phi=\beta / \alpha+\beta^{\prime} / \alpha^{\prime} .
\end{aligned}
$$

The fact $\alpha / 1+\alpha^{\prime} / 1=\left(\alpha+\alpha^{\prime}\right) / 1$ is obvious.

As Hilton pointed out [2], even in the abelian case and with $D$ the 
class of epics, the extended addition is not distributive. At least with $\delta \in \mathbb{C}$ we have

$$
(\delta / 1)\left(\beta / \alpha+\beta^{\prime} / \alpha^{\prime}\right)=(\delta / 1)(\beta / \alpha)+(\delta / 1)\left(\beta^{\prime} / \alpha^{\prime}\right),
$$

since, with the notation above,

$$
\begin{aligned}
(\delta / 1)\left(\beta / \alpha+\beta^{\prime} / \alpha^{\prime}\right) & =(\delta / 1)\left(\beta \phi+\beta^{\prime} \psi\right) / \alpha \phi=\delta\left(\beta \phi+\beta^{\prime} \psi\right) / \alpha \phi \\
& =\left(\delta \beta \phi+\delta \beta^{\prime} \psi\right) / \alpha \phi=\delta \beta / \alpha+\delta \beta^{\prime} / \alpha^{\prime} \\
& =(\delta / 1)(\beta / \alpha)+(\delta / 1)\left(\beta^{\prime} / \alpha^{\prime}\right) .
\end{aligned}
$$

Let us compare

$$
(\delta / \gamma)\left(\beta / \alpha+\beta^{\prime} / \alpha^{\prime}\right), \quad(\delta / \gamma)(\beta / \alpha)+(\delta / \gamma)\left(\beta^{\prime} / \alpha^{\prime}\right)
$$

in the general case. Again $\beta / \alpha+\beta^{\prime} / \alpha^{\prime}=\left(\beta \phi+\beta^{\prime} \psi\right) / \theta$. Let

$$
\downarrow \xi^{\prime}, \xi, \gamma, \beta \phi+\beta^{\prime} \psi \downarrow
$$

and then the left side is $\delta \xi^{\prime} / \theta \xi$. To compute the right-hand side, let $\downarrow s, t, \gamma, \beta \downarrow$ and $\downarrow s^{\prime}, t^{\prime}, \gamma, \beta^{\prime} \downarrow$; and then we have to construct the sum $\delta s / \alpha t+\delta s^{\prime} / \alpha^{\prime} t^{\prime}$. But here we encounter a question of existence since, having $\downarrow x^{\prime}, x, \alpha^{\prime} t^{\prime}, \alpha t \downarrow$, we need the sum $\delta s x+\delta s^{\prime} x^{\prime}$ in $\mathcal{C}$. We have $\alpha t x=\alpha^{\prime} t^{\prime} x^{\prime}$; hence there is a $\lambda$ satisfying $t x=\phi \lambda, t^{\prime} x^{\prime}=\psi \lambda$. By our assumptions $\beta \phi \lambda+\beta^{\prime} \psi \lambda=\beta t x+\beta^{\prime} t^{\prime} x^{\prime}=\gamma s x+\gamma s^{\prime} x^{\prime}$ is defined. Here we need an additional assumption, and this case could be, for instance, the assumption of Kurosh et al. [5] that for $\gamma$ monic, if $\gamma f+\gamma g$ is defined then $f+g$ is defined. Assuming this we have $\delta s x+\delta s^{\prime} x^{\prime}$ defined, provided that $\gamma$ is monic. Then, by $\left(\beta \phi+\beta^{\prime} \psi\right) \lambda=\gamma\left(s x+s^{\prime} x^{\prime}\right)$, there is a $\mu$ for which $\xi \mu=\lambda, \xi^{\prime} \mu=s x+s^{\prime} x^{\prime}$, hence

$$
(\delta / \gamma)(\beta / \alpha)+(\delta / \gamma)\left(\beta^{\prime} / \alpha^{\prime}\right)=\delta \xi^{\prime} \mu / \alpha \phi \xi \mu,
$$

whereas $(\delta / \gamma)\left(\beta / \alpha+\beta^{\prime} / \alpha^{\prime}\right)=\delta \xi^{\prime} / \alpha \phi \xi$. Unfortunately, in the general case $\mu \notin D$, otherwise we would have at least left distributivity for $\delta / \gamma$ with $\boldsymbol{\gamma}$ monic.

Let us compare

$$
\left(\beta / \alpha+\beta^{\prime} / \alpha^{\prime}\right)(\delta / \gamma), \quad(\beta / \alpha)(\delta / \gamma)+\left(\beta^{\prime} / \alpha^{\prime}\right)(\delta / \gamma) .
$$

Assume that the right-hand side is defined; so if $\downarrow v, u, \delta, \alpha \downarrow$, then $(\beta / \alpha)(\delta / \gamma)=\beta u / \gamma v$ and similarly $\left(\beta^{\prime} / \alpha^{\prime}\right)(\delta / \gamma)=\beta u^{\prime} / \gamma v^{\prime}$, thus with $\downarrow y^{\prime}, y, \gamma v^{\prime}, \gamma v \downarrow$ we have $\beta u y+\beta^{\prime} u^{\prime} y^{\prime}$ defined in $\mathcal{C}$. If the left side is defined, then with $\downarrow \psi, \phi, \alpha^{\prime}, \alpha \downarrow$ we have $\beta / \alpha+\beta^{\prime} \alpha^{\prime}=\left(\beta \phi+\beta^{\prime} \psi\right) / \theta$, $\theta=\alpha \phi=\alpha^{\prime} \psi$. Therefore, with $\downarrow \eta, \xi, u, \phi \downarrow,\left(\beta / \alpha+\beta^{\prime} / \alpha\right)(\delta / \gamma)$ $=\left(\beta \phi+\beta^{\prime} \psi\right) \xi / \gamma v \eta$. Since $\alpha \phi=\alpha^{\prime} \psi$, there is a pullback $\downarrow \eta^{\prime}, \xi, u^{\prime}, \psi \downarrow$ 
such that $v^{\prime} \eta^{\prime}=v \eta$. So $\gamma v \eta=\gamma v^{\prime} \eta^{\prime}$ and a $\mu$ exists such that $y \mu=\eta$, $y^{\prime} \mu=\eta^{\prime}$, hence

$$
\left(\beta / \alpha+\beta^{\prime} / \alpha^{\prime}\right)(\delta / \gamma)=\left(\beta u \eta+\beta^{\prime} u^{\prime} \eta^{\prime}\right) / \gamma v \eta=\left(\beta u y+\beta^{\prime} u^{\prime} y^{\prime}\right) \mu / \gamma v \eta
$$

and $\gamma v \eta=(\gamma v y) \mu=\left(\gamma v^{\prime} y^{\prime}\right) \mu$. Again $\mu$ is not necessarily in $D$, otherwise we could have right distributivity.

VIII. In the particular case $\mathbb{D}=\mathfrak{C}$, assuming that the involved sums are defined, we have both right- and left-distributivity.

IX. In the bicategorical case $(D=\$)$, assuming that the involved sums are defined, we have at least inequalities

$$
\begin{aligned}
{[S]\left([R]+\left[R^{\prime}\right]\right) } & \geqq[S][R]+[S]\left[R^{\prime}\right] ; \\
{[R][T]+\left[R^{\prime}\right][T] } & \geqq\left([R]+\left[R^{\prime}\right]\right)[T],
\end{aligned}
$$

for relations in $R_{e}$.

\section{REFERENCES}

1. A. Klein, Relations in categories, Illinois J. Math. 14 (1970), 536-550.

2. P. Hilton, Correspondences and exact squares, Proc. Conference on Categorical Algebra (La Jolla, Calif., 1965), Springer, New York, 1966, pp. 254-271. MR 34 \#4326.

3. B. Mitchell, Theory of categories, Pure and Appl. Math., vol. 17, Academic Press, New York, 1965. MR 34 \#2647.

4. J. Isbell, Natural sums and abelianizing, Pacific J. Math. 14 (1964), 1265-1281. MR 31 \#3478.

5. A. G. Kuroš, A. H. Livšic and E. G. Sul'gelfer, Foundations of the theory of categories, Uspehi Mat. Nauk 15 (1960), no. 6 (96), 3-52=Russian Math. Surveys 15 (1960), 1-46. MR 23 \#A1688; MR 22 \#9526.

Case Western Reserve University, Cleveland, Ohio 44106

Bar-Ilan University, Ramat-GaN, Israel 